\title{
VOLVER A INTENTARLO: PROYECTO EDUCATIVO-LABORAL DE JOVENES ADULTOS SOCIALES
}

\author{
LEANDRO SEPÚLVEDA V.
}

\begin{abstract}
RESUMEN
En su aspecto medular, el artículo pretende recoger e interpretar las principales representaciones del mundo del trabajo y el proyecto educativo-laboral que realizan jóvenes adultos sociales que se encuentran actualmente cursando su enseñanza media en el sistema de educación de adultos. En este texto se pretende entregar antecedentes empíricos de un segmento social juvenil que, habiendo abandonado la educación formal, después de algunos años, intenta terminar sus estudios secundarios en un sistema alternativo en búsqueda de mejores posibilidades de inserción social.

El artículo sintetiza algunas hipótesis conclusivas que remiten al perfil general de estos jóvenes, su experiencia educativa y laboral, las representaciones generales que realizan de su entorno y su proyección de futuro personal. En términos generales se señala que estos jóvenes expresan una nueva forma de moratoria y son portadores de un proyecto educativo no exento de desajuste en sus expectativas. Carentes de redes sociales significativas, participando en un modelo de formación precario y con bajos niveles de innovación, en su discurso, con todo, prevalece un visión optimista de futuro y de confianza en su propio esfuerzo para salir adelante.

Tales consideraciones parecen relevantes en una discusión amplia sobre el futuro del sistema de educación de adultos, en perspectiva de favorecer propuestas formativas más adecuadas para hacer frente a las transformaciones del mundo del trabajo, diversificando un modelo extremadamente rígido de formación académica pro-universitaria y poco funcional al perfil del joven que motiva el interés de este estudio.
\end{abstract}

PALABRAS CLAVE: JÓVENES, EDUCACIÓN Y TRABAJO

* Antropólogo Social; investigador del Centro de Investigación y Desarrollo de la Educación (CIDE); docente del Magíster en Políticas Sociales y Desarrollo Local, Universidad ARCIS y Escuela de Servicio Social, Universidad Católica de Valparaíso. E-Mail: 1sepulve@cide.cl. 


\section{RESUMO}

Em seu aspecto primordial, o projeto pretende recolher e interpretar as principais representações do mundo do trabalho e o projeto de educação e trabalho que são realizados por jovens adultos sociais que atualmente estão cursando o ensino médio no sistema de educação para adultos. Neste texto pretende-se entregar antecedentes empíricos de um segmento social juvenil que tendo abandonado a educação formal, depois de alguns anos, tenta terminar seus estudos secundários (ensino médio) em um sistema alternativo em busca de melhores possibilidades de inserção social.

$\mathrm{O}$ artigo sintetiza algumas hipótesis conclusivas que remetem ao perfil geral destes jovens, sua experiência educativa e de trabalho, as representações gerais que fazem de tudo a sua volta e sua projeção pessoal de futuro. Em termos gerais é possível ver que estes jovens expressam uma nova forma de moratória e são portadores de um projeto educativo não isento de desajustes em suas expectativas. Carentes de redes sociais significativas, participando em um modelo de formação precário e com baixos níveis de inovações, em seu discurso, contudo, prevalece uma visão otimista de futuro e de confiança em seu próprio esforço para seguir adiante.

Tais considerações parecem relevantes em uma discussão ampla sobre o futuro do sistema de educação de adultos, em perspectiva de favorecer propostas formativas mais adequadas que façam frente às transformações do mundo do trabalho, diversificando um modelo extremamente rígido de formação acadêmica pro-universitária e pouco funcional ao perfil do jovem que motiva o interesse deste estudo.

PALAVRAS CHAVE: JOVENS, EDUCAÇÃO E TRABALHO

\section{ABSTRACT}

In its medullar aspect, this article tries to collect and interpret the main representations of the working world and the labor-educational project involving social adultyoung people that are currently coursing their secondary schooling in the educational system for adults. In this text, we seek to offer empiric antecedents of a juvenile social segment that - having left the formal education, after a couple of years - is trying to finish its secondary education in an alternative system, in search for better opportunities of social insertion.

The present article synthesizes some conclusive hypotheses referring to the general profile of these young individuals, their educational and work experience, the global representations of their environment and the projections referring to their own future. In general, we point out that these young persons express a new form of moratorium, in addition to be carriers of a new educational project, not without some displacement in their expectations. These are young people lacking of significant social networks, participating in a pattern of precarious formation, with low innovative levels in their discourse. Nevertheless, it prevails in them an optimistic vision of the future and a sense of trust in their own effort to make it through.

Such considerations seem to be relevant in a broad discussion about the future of the educational system for adult people, in perspective of favorable formative proposals that can be adequate in order to face the transformations of the working world, diversifying an extremely rigid pattern of pro-university academic formation that is little functional to the profile of the young individual that is the main subject of this study.

KEY WORDS: YOUNG PEOPLE, EDUCATION AND WORK 


\section{INTRODUCCIÓN}

DESDE HACE ALGUNOS AÑOS el sistema de educación de adultos en Chile ha tenido un proceso de transformaciones más que relevante. En efecto, en la última década se ha verificado un crecimiento sostenido de su matrícula, incrementando en más de 50 mil plazas el total de alumnos entre los años 1990 y 2000 . Actualmente, con una tasa de crecimiento anual de la matrícula superior al 7,5\%, el sistema acoge un número cercano a las 120 mil personas, y de este total, el 75\% corresponde a jóvenes de ambos sexos entre 15 y 24 años. ${ }^{1}$

Este cuadro representa una modificación importante de la imagen tradicional de la educación de adultos en nuestro país, por lo general asociada a un modelo de nivelación de estudios para personas mayores, insertas en el sistema laboral y bajo la modalidad de formación vespertina. A diferencia de eso, hoy el grueso de los estudiantes de educación de adultos corresponde a jóvenes pobres de ambos sexos, desertores o expulsados del sistema educacional tradicional, y que retornan a sus estudios luego de algunos años fuera del sistema. ${ }^{2}$

Se trata de jóvenes con una experiencia laboral fragmentaria, muchos de ellos dependientes de su grupo familiar, y con una amplia disponibilidad horaria para el desarrollo de su actividades formativas. Este perfil, denominado joven adulto social, sintetiza una situación de indefinición y/o prolongación del período de construcción de identidad del sujeto, combinando actividades propiamente estudiantiles con actividades laborales y otras responsabilidades familiares. En rigor, correspondería a una categoría social específica de la heterogénea realidad juvenil actual, íntimamente vinculada a las profundas transformaciones económicas y socioculturales de los últimos decenios.

En efecto, la alta presencia de jóvenes desertores recientes del sistema tradicional al interior de los establecimientos de educación de adultos, parece reflejar un hecho significativo, esto es, la incidencia de la presión social por una mayor escolarización, y el reconocimiento

1 Ministerio de Educación, Coordinación Nacional de Educación de Adultos: «Orientaciones 2001 para la educación de personas jóvenes y adultas». Santiago.

2 Por cierto, este hecho ha sido advertido por las autoridades educacionales en Chile. Durante la década del noventa se creó la figura de los Centros de Educación Integrada de Adultos para adecuar la oferta formativa a la nueva demanda, y en la actualidad, el Programa Chile Califica representa un esfuerzo destinado a coordinar esfuerzos educativos con una mayor vinculación al mundo del trabajo. 
extendido de que la certificación de educación secundaria completa es una condición básica para la incorporación al mercado de trabajo.

El abandono temprano de la educación secundaria restringe cualquier intento de proyecto alternativo. Con serias limitaciones de inserción y permanencia en un empleo, estos jóvenes reconocerían la necesidad imperiosa de contar con la licencia de educación secundaria, encontrando en el establecimiento de educación de adultos una oportunidad para volver a intentarlo.

Aunque los motivos esgrimidos para concurrir a un centro de estas características pueden ser variados, los antecedentes disponibles reflejan una gran homogeneidad socioeconómica de sus integrantes. Se trata de jóvenes en situación de exclusión social, por lo general, expulsados o desertores del sistema tradicional de educación y depositarios de una serie de problemas sociales que dificulta su permanencia en un establecimiento formal y su incorporación al trabajo.

Son jóvenes que tienen como perspectiva inmediata (independientemente de sus intenciones o deseos) la vinculación al mundo laboral, pero que carecen de los recursos sociales básicos para desarrollar una estrategia con algún nivel de éxito en el corto plazo.

Este artículo pretende destacar algunos resultados de un estudio enfocado en este segmento de la realidad social. El estudio estuvo orientado a recoger e interpretar las principales representaciones del mundo del trabajo y el proyecto educativo-laboral que realizan estos jóvenes. $^{3}$

El foco de interés de la investigación resaltaba la importancia de concentrar los esfuerzos interpretativos en los sentidos, expectativas y proyectos que organizan y re-organizan la vida personal de los sujetos. ¿Qué razones llevan a estos jóvenes, de manera creciente, a intentar una nueva experiencia educacional destinada a completar su enseñan-

3 En su fase empírica, el estudio consistió en la aplicación de una entrevista estructurada a una muestra de 381 estudiantes provenientes de 18 establecimientos educacionales de adultos en la Región Metropolitana que imparten alguna modalidad de la enseñanza media (científico humanista 2 en 1 , científico humanista adultos y técnico profesional adultos). El 47,5\% de los encuestados son hombres y el 52,5\% mujeres, con un promedio de edad de 19 años. Junto a esto, se realizaron 10 entrevistas en profundidad a jóvenes que estuviesen cursando algún nivel de formación secundaria a nivel de adultos, con el objetivo de profundizar en el perfil general de los jóvenes, sus antecedentes familiares, existencia y nivel de densidad de las redes de apoyo social, trayectoria educacional, experiencia laboral, representación del mundo del trabajo, así como expectativas y proyecto educativo laboral en el futuro inmediato. 
za media?; ¿cuál es su experiencia familiar inmediata y qué motivos se esgrimen como causa de su fracaso escolar anterior?, ¿cuál es su experiencia laboral y qué proyecciones realizan en relación al mundo del trabajo?; en un sentido más general, ¿cuáles son los sueños y expectativas en el futuro inmediato de estos jóvenes? Éstas son algunas de las preguntas que orientaron esta investigación.

De un modo general, el estudio enfatiza el análisis de los sentidos atribuidos al trabajo, la incorporación a un empleo, y el valor que se asigna a la formación recibida en el marco de la permanencia en el sistema escolar. Con esto, se pretende entregar información empírica sobre la relación educación y trabajo, discusión que en los últimos años ha estado focalizada, mayormente, en el análisis de los sistemas y sus distintos componentes (y con un fuerte sesgo prescriptivo), en desmedro de los sujetos y sus prácticas, los procesos de adecuación al orden cambiante, y las re-elaboraciones colectivas de sentido bajo las nuevas exigencias de tipo estructural.

Menos que un intento por abordar grandes procesos sociales o asumir en su complejidad las transformaciones de los sistemas educativo o laboral, este estudio se intenta situar en el marco de la tradición antropológica destinada a hacerse cargo de ámbitos acotados de la realidad sociocultural o la vida interior de las sociedades, ${ }^{4}$ relevando la figura de actores específicos, sus experiencias de vida, sus representaciones y proyecciones como un insumo fundamental para una discusión informada.

Sostenemos que el reconocimiento de los procesos vivenciados por sujetos particulares en un contexto social de grandes transformaciones, representa un esfuerzo conducente menos que a entregar respuestas, formular preguntas adecuadas que, junto a otras, oriente un posicionamiento productivo en el pensamiento social de este tiempo. Como señala Maffesoli, éste es un tiempo «en el cual, debido al cambio de valores que se lleva a cabo, es necesario saber escuchar como crece la hierba» (Maffesoli, 1993:24).

En lo que sigue se presenta un breve marco de antecedentes generales destinado a resaltar las paradojas del actual momento de la modernización de nuestra sociedad para las generaciones jóvenes; esto es, la fuerte incidencia de la escolarización y la preeminencia de un mo-

4 Balandier destaca la importancia de asumir «la vida al interior de las sociedades, la que se descubre debajo de las apariencias institucionales, de sus formas y fórmulas oficiales» (Balandier, 1975:245). 
delo de educación secundaria en el construcción de proyecto personal, junto a la creciente segmentación social derivada de un modelo productivo caracterizado por la precariedad, la dualización y la integración precaria. Se sostendrá que, justamente, éste es el contexto económico social que engloba la actual situación de los jóvenes objeto de nuestro interés.

En la segunda parte del artículo se entrega algunos de los resultados principales de la indagación empírica, particularmente a partir de los resultados obtenidos de una encuesta representativa a jóvenes estudiantes en el sistema de educación de adultos en la Región Metropolitana. El artículo concluye con algunas observaciones generales derivadas del estudio y que pueden ser de utilidad para repensar las propuestas formativas a nivel de educación de adultos en el marco de sus actuales características.

\section{ANTECEDENTES GENERALES}

\section{Jóvenes en situación de exclusión social. La tensión entre el proceso de escolarización y la marginación del mercado laboral}

Es un hecho consensual que a lo largo de la década del noventa se ha producido un conjunto de transformaciones de gran magnitud al interior del sistema educacional chileno. En breve, durante este período, se observa i) la consolidación del proceso de amplia cobertura de la matrícula básica y media alcanzando, en este último caso, al $85 \%$ de la población en edad escolar, ii) a nivel institucional se implementa un ambicioso programa de reforma curricular, enfatizando en principios de formación general, funcionales a los requerimientos del mundo del trabajo y nuevas formas de organización de la vida social moderna, y iii) se enfatiza en el desarrollo de nuevos contextos de aprendizaje, aportando recursos institucionales para mejorar las bases materiales de estas iniciativas (Cox, 2001:283).

La reforma educacional impulsada durante estos últimos años, particularmente en lo que refiere a la educación secundaria, ha intentado responder la pregunta de cómo vincular el sistema educativo al conjunto de requerimientos del mundo actual, favoreciendo una mayor igualdad de oportunidades sociales y ampliando las alternativas formativas con la intención de evitar las tendencias a la segmentación social (Belleï, 2000:45). 
Tales esfuerzos se vinculan, sin duda, a las nuevas formas de organización y demandas del mundo productivo, sustentado mayormente en la organización del conocimiento, las relaciones interpersonales y la capacidad de adaptación a exigencias cambiantes, en el marco de un sistema laboral crecientemente desregulado.

Como consecuencia de este proceso, en nuestra sociedad existe una amplia valoración de la educación para la inserción en el mundo del trabajo y la elaboración de un proyecto de vida, reconociéndose que la educación secundaria más que una meta, constituye una base mínima de acceso al mundo laboral. ${ }^{5}$

El incremento de las tasas de escolarización (potenciado por el proceso de reforma educacional durante la década del noventa) ha servido como principal argumento para el augurio de un futuro de mayor bienestar de las nuevas generaciones: «son varias las razones que permiten afirmar que la globalización, así como la creciente ampliación de fronteras de competitividad en un escenario de acelerada incorporación de innovaciones tecnológicas, se acompaña de una notable elevación de potencialidades de la contribución de los jóvenes al desarrollo de sus sociedades. Ciertamente, el principal de estas razones es el papel destacado del conocimiento como motor de las transformaciones y como recurso fundamental de las sociedades para enfrentar los desafíos que ellas les plantean» (CELADE: 2000:14). A comienzos de la década del noventa ésta fue la afirmación más socorrida para fortalecer una visión optimista de futuro. ${ }^{6}$

5 Sin embargo, independientemente de los esfuerzos institucionales realizados, de los logros alcanzados y, muy particularmente, del grado de consolidación de un sentido común acerca de la importancia de contar con la escolaridad completa para hacer frente a los desafíos de la integración, el sistema educacional chileno también refleja, de un modo pertinaz, las hondas diferencias sociales y que inciden negativamente en los sectores más pobres. En efecto, hacia el año 2000 más del 75\% de los jóvenes que desertan del sistema educacional en Chile corresponden a los dos deciles de menores ingresos. En los establecimientos educacionales municipalizados, el tiempo de egreso promedio alcanza casi a los 6 años, frente a los 4,4 de los establecimientos particular pagado, y el porcentaje de egreso entre ambos tipos de establecimientos presenta una diferencia cercana a los 30 puntos porcentuales. De este modo, si a fines de la década del noventa un tercio de los alumnos de la enseñanza secundaria no culminaba su trayectoria de formación, el grueso de ellos correspondía a jóvenes de estratos socioeconómicos pobres.

6 Véase, por ejemplo, CEPAL: Transformación productiva con equidad, Santiago, 1990. 
Sin embargo, entrando a la mitad de esta nueva década, los análisis señalan que, pese a los nuevos estilos de desarrollo y la preeminencia de un modelo productivo fundado en la sociedad del conocimiento, las nuevas generaciones jóvenes, supuestamente llamadas a integrarse masivamente en este nuevo escenario, vivencian la situación de desempleo estructural que ha caracterizado la situación en América Latina durante los últimos decenios: «el desempleo juvenil tiene características netamente estructurales, y ha persistido en niveles sumamente elevados en los últimos cuarenta años (al menos), tanto en épocas de crisis como en etapas de crecimiento económico sostenido. Los comportamientos corporativos de empresarios y trabajadores organizados jamás han priorizado la incorporación laboral de jóvenes, y las políticas públicas de empleo han concentrado tradicionalmente sus acciones en el jefe de hogar (adulto, hombre), en el marco de modelos de desarrollo y estructuras familiares que ya no son hegemónicas en nuestras sociedades» (Rodríguez, 2000).

El caso chileno es una buena expresión del cuadro antes reseñado; la tasa de desempleo juvenil ha variado de manera proporcional a la evolución de la tasa general, manteniendo, de manera constante, una brecha en relación a lo observado para el conjunto de la fuerza de trabajo.

Por cierto, en los últimos años es mayor el porcentaje de jóvenes que posterga su incorporación a la fuerza de trabajo debido a su permanencia en el sistema educacional, sin embargo, este hecho no inhibe lo anterior e incluso se agudiza en el caso de los jóvenes más pobres. En efecto, a esta situación constante de altas tasas de desempleo juvenil, es necesario agregar la diferencias significativas si se analiza la situación por grupos socioeconómicos. Este rasgo está íntimamente asociado a la desigual distribución del capital económico y cognitivo de la población, produciéndose una suerte de círculo vicioso que las políticas de Estado y los esfuerzos en el área educacional difícilmente pueden hacer frente.

Como resultado, son los jóvenes más pobres quienes llevan el peso de la situación de desempleo. En el caso chileno, la Encuesta CASEN 2000 señala que el desempleo juvenil afecta mayoritariamente a los jóvenes del $20 \%$ de menores ingresos de la población, con tasas en torno al 44,6\% para jóvenes de entre 15 a 19 años y de $38,1 \%$ para jóvenes de 20 a 24 años. De igual manera, el desempleo afecta más fuertemente a vendedores de tiendas, trabajadores agropecuarios, obreros y jornaleros y personal de servicio doméstico joven. Los profesionales en este tramo de edad presentan, comparativamente, un bajo desempleo. 
La situación laboral de los jóvenes no puede independizarse de la tendencia general al deterioro de la calidad de las ocupaciones y el crecimiento de los empleos de baja productividad. El incremento de la informalidad o el trabajo precario, ${ }^{7}$ es una señal del aumento de la polaridad social, y los jóvenes, en este sentido, son un sector particularmente sensible a su efecto en todos los ámbitos de la realidad social.

La situación educacional y socioeconómica de origen son los principales factores asociados a la desigual inserción y permanencia en puestos de trabajo. Los jóvenes que no completan más de 8 años de educación se desempeñan, en su mayor parte, en puestos de trabajo precarios, y con ingresos mensuales insuficientes para garantizar el bienestar de su grupo familiar. En el caso de jóvenes con 12 o más años de estudio, los ingresos se elevan por sobre el triple de los anteriores. ${ }^{8}$

Menos que un mejoramiento de la situación económica y social, en los últimos años se ha vivenciado un ahondamiento de las condiciones de desigualdad y polarización social. El sector juvenil, y muy particularmente los jóvenes de escasos recursos, son quienes han sufrido el mayor impacto de este proceso. La fragmentación del mundo del trabajo, el impacto de la flexibilización y la condición de exclusión parcial o absoluta del empleo formal constituye un cuadro de características estructurales que la expansión del sistema educacional no ha podido revertir. ${ }^{9}$

7 Entre 1990 y 1996 el empleo desprotegido en Chile había aumentado de un $17 \%$ al $22.3 \%$. Véase Rodrik, 2001.

8 Los esfuerzos destinados a incrementar los años de escolaridad de la población joven se ven limitados por el proceso de devaluación de la educación, tal como se destaca en los análisis de CEPAL: «conjuntamente con el aumento de la cobertura y del nivel educacional promedio en la sociedad, se produce una devaluación de la educación por cuanto se necesitan más años de estudio para lograra una misma inserción ocupacional y un ingreso equivalente. Por ello, existe superación educativa intergeneracional si la educación alcanzada por los jóvenes en la generación presente es mayor que la de sus padres, descontando dicha devaluación. Pero cuanto menor es la educación de los padres, mayor es el número de años adicionales en educación que requieren los hijos para lograr ingresos más elevados que sus progenitores [...] Las condiciones intergeneracionales que configuran este cuadro de limitaciones al bienestar, quedan de manifiesto cuando se comprueba que, junto a la notable ampliación de la cobertura de la educación, persiste una escasa proporción de jóvenes que superan el nivel educacional de sus padres, considerada la devaluación» (CEPAL, 2000:104).

9 Como podrá verse, la mayoría de los jóvenes objeto de nuestro interés intenta finalizar la enseñanza media porque, en rigor, no tienen mayores alternativas al ser marginados del mercado laboral. 
En resumen, el período que contempla los últimos años de la década del noventa y el comienzo de un nuevo siglo, entrega señales paradójicas respecto a la situación de la población joven; por una parte, la evolución de las tasas de crecimiento poblacional y los esfuerzos nacionales por incrementar la escolaridad de la población constituyen bases optimistas respecto a la situación general de los jóvenes y su proyección hacia futuro.

Sin embargo, las características de la evolución de la actividad productiva, el comportamiento del empleo en el marco de las transformaciones económicas propias de esta globalización, y la inserción laboral de las nuevas generaciones en esta última década, entrega señales negativas y que se agudizan bajo una mirada del aumento de la brecha social en nuestro país y el resto del continente.

\section{Conductas juveniles y sentido de la educación y el trabajo}

Estos antecedentes permiten relevar la complejidad del campo en que se desenvuelve la problemática juvenil. En efecto, puede sostenerse que en la actualidad se verifica un proceso de desarrollo social que presenta características de inclusión/exclusión y que se expresa, con igual nivel de paradoja, en el comportamiento sociocultural de las generaciones más jóvenes.

Desde nuestro punto de vista, las manifestaciones culturales de la juventud, menos que dar cuenta de una ruptura con el sistema vigente, contienen esta contradicción fundamental, ratificada en la incidencia de la escolarización en la historia de vida de la gran mayoría de la población (y muy particularmente de las nuevas generaciones). Un énfasis en esta dirección obliga a una conceptualización menos rígida respecto a la cultura juvenil que la desarrollada en los años precedentes.

En efecto, una de las tesis predominantes durante la década de los ochenta para la caracterización de la realidad juvenil resaltaba el impacto desintegrador del proceso de modernización económica, incidiendo en la emergencia de identidades segmentadas y comportamientos de tipo anómicos.

Bajo esta perspectiva, los estudios de la década del ochenta (donde destacan los trabajos de Martínez y Valenzuela) caracterizan a la juventud, y muy particularmente, la juventud de los estratos socioeconómicos pobres, como un sector sometido a un fuerte proceso de desintegración cultural, verificándose la emergencia de conductas rupturistas y disfuncionales al orden social establecido. La juventud urbana 
marginal, ${ }^{10}$ irrumpe como una masa excluida de los mecanismos de movilidad e integración social, y participando de una «anomia subjetiva» que es correspondiente a una percepción colectiva que señala que la sociedad no ofrece alternativa de desarrollo e integración. La violencia o el consumo de drogas de los jóvenes, desde esta perspectiva, es una respuesta propia de una situación de ausencia de reglas y normas socialmente compartidas (Espinoza et al., 2000:14).

Este modelo analítico, ampliamente desarrollado durante las dos décadas pasadas para caracterizar el sujeto juvenil urbano de las grandes ciudades de América Latina, presenta una serie de deficiencias contrastadas con la evidencia empírica. ${ }^{11}$ En efecto, los estudios sobre el comportamiento cultural de la juventud, han demostrado que actitudes como la apatía, la desvinculación de la política, la conducta violenta o el consumo de drogas, no constituyen rasgos asociados, necesariamente, a la situación de pobreza sostenida, verificándose de hecho conductas heterogéneas en diversos estratos socioeconómicos. De igual modo, el alto índice de retención escolar y la adscripción a un modelo de consumo que expresan los estudios de opinión de jóvenes en los últimos años, cuestionan la centralidad de la tesis anómica.

Como señala Espinoza, en su formulación, el problema de este modelo analítico fue intentar asociar exclusión y anomia; el primer concepto hace referencia al bloqueo de las vías legítimas de movilidad social, lo que no involucra, necesariamente, dejar de compartir orientaciones culturales del modelo de desarrollo predominante; la anomia, por el contrario, involucra la pérdida de sentido de las orientaciones culturales, lo que lleva a una desorganización social radical.

Para este autor, «los jóvenes de los noventa aparecen preocupados de su futuro y actuando consecuentemente para mejorar su posición. En su mayoría, los jóvenes chilenos muestran una alta disposición a la integración social; reconocen claramente que se encuentran en un mundo donde el acceso a oportunidades les demanda esfuerzo. Lejos de desalentarlos su comportamiento revela que legitiman la

10 Algunos autores utilizaron el concepto de sub-proletarización para dar cuenta de esta realidad; véase Weinstein, 1988.

11 Sin ánimos de mayor desarrollo, una de las dificultades tiene que ver con la confluencia analítica del contexto social y político - ausencia de democracia, regímenes autoritarios-, y la revisión de la acción de un actor y sus procesos de identidad. La tesis de Valenzuela, de hecho, radica en la necesidad de recomponer el orden social caracterizado por la «anomia objetiva» para un necesario reencauzamiento de la acción de los sujetos. Véase Valenzuela, 1984. 
integración social y valoran la educación formal como el mecanismo a través del cual ésta se alcanza [...] Más individualistas que en generaciones anteriores, ponen mayor énfasis en su educación formal porque reconocen en la calificación y el conocimiento la clave para una integración social exitosa...».

La extendida valoración subjetiva de los mecanismos de integración convive con una situación evidente de exclusión social que afecta a una masa importante de jóvenes pobres, pese a ello, «los datos disponibles muestran que muchos jóvenes legitiman la integración a pesar de encontrarse en una situación de exclusión» (Ibídem:16).

Este diagnóstico sobre la subjetividad juvenil difiere de otros análisis existente en el marco de la discusión latinoamericana. Para celade, la creciente incapacidad del mercado laboral por incorporar a los jóvenes con bajas calificaciones, y las dificultades que enfrentan los Estados por reformar la educación y los sistemas de capacitación a un ritmo ajustado a los requerimientos del sistema productivo, implican el ahondamiento de un hueco normativo en nuestras sociedades que hace de los jóvenes de los sectores populares sujetos de marginación.

Aunque se reconoce que los jóvenes tienen una alta participación simbólica en la sociedad que moldea sus aspiraciones, «la combinación de estos elementos contribuye a la formación de subculturas marginales, pandillas y barras, que generan sus propios códigos y están fuertemente expuestas a la incorporación de los hábitos y comportamientos emergentes de las situaciones de marginalidad y exclusión social» (CELADE, 2000:20). Desde esta perspectiva, los problemas estructurales de segmentación y exclusión tienen un correlato de tipo cultural que agudiza la diferenciación y consolida un cuadro de anomia estructural.

En esta argumentación el modelo conceptual de tribus juveniles urbanas, emerge como la figura analítica más socorrida; pese a ello, no parece existir un consenso entre quienes trabajan este concepto en la realidad latinoamericana acerca del grado de autonomización, aislamiento y ruptura con los mecanismos sociales de integración (la familia, la escuela, el trabajo) de estas particularidades formas de agrupación. $^{12}$

12 Una lectura que relativiza el impacto estructural de la figura de las tribus lo entrega Silva: «las tribus, responden no sólo a una cuestión estética - aunque es innegable su componente y despliegue - sino, por sobre todo, a una respuesta ingeniosa y circunstancial que, algunos jóvenes, dan al estado actual de cosas, que las sociedades contemporáneas les ofrecen. Por otra parte, salvo algunas 
Desde nuestro punto de vista, esta distinción conceptual puede ser relevante en el análisis de las transformaciones que viven las generaciones jóvenes y que se caracteriza por las paradojas antes reseñadas, sin embargo, la expresión cultural de esta realidad parece más lejana de la figura de comportamiento anómico aquí revisado.

La tendencia de la orientación cultural de los sujetos parece ser mayormente orientada en una lógica que expresa las contradicciones anotadas que a una ruptura y pérdida de sentidos sociales generales. ${ }^{13}$ La rigurosidad conceptual obliga, bajo este punto de vista, a concebir la realidad juvenil y muy particularmente la de la mayoría de los jóvenes pobres, como una situación propiamente de exclusión social.

En efecto, el concepto de exclusión social refiere tanto a una dimensión material como también a situaciones de precaria inserción institucional y cultural al interior de una sociedad. En su tratamiento reciente, el concepto da cuenta de un «adentro/afuera, que es materia de definición y que no debe ser entendido como no pertenencia social, sino como una falta de acceso a las oportunidades y beneficios que genera el sistema ${ }^{14}$ De este modo, esta falta de acceso a las oportunidades y beneficios que genera el sistema no depende solamente de la voluntad o intereses de las personas, sino también de las formas institucionales y de la dinámica de transformación del propio sistema social» (Wormald y Ruiz-Tagle, 1999:75).

Este enfoque, creemos, introduce una dimensión fundamental para la distinción analítica en el tratamiento de la realidad juvenil en la actualidad. Culturalmente - la influencia del sistema educacional es, en este sentido, innegable_- ${ }^{15}$ los jóvenes expresan un sentido de

excepciones, las tribus tienen una clara connotación temporal y fugaz, sobre todo en lo que se refiere a la permanencia de sus miembros en tales agrupaciones» (Silva, 2002:118).

13 Es decir, los procesos de individualización y desafiliación, menos que expresiones subculturales de generaciones que se desvinculan de un orden divergente, constituyen las bases precarias de organización del sentido de vida; los jóvenes son, desde este punto de vista, uno más de los actores de un sistema social general precario.

14 El destacado es nuestro.

15 Una expresión evidente de lo anterior se encuentra en la Encuesta Nacional a los Actores del Sistema Educativo 2004 del CIDE, ante la alternativa de establecer un pronóstico de su experiencia educativa, un $72.1 \%$ de los alumnos de los diversos establecimientos educacionales chilenos manifestaron la decisión de terminar estudios de educación superior, ya sea en Universidades, Institutos Profesionales o Centros de Formación Técnica. Esta orientación se distribuye en un $74 \%$ de estudiantes de establecimientos municipalizados, un $85 \%$ de esta- 
integración y reconocimiento de mecanismos de participación social. Sin embargo, de igual manera, su identidad está cruzada por el reconocimiento de los procesos de diferenciación y exclusión social, con una fuerte marca de las condiciones y posibilidades que ofrece la certificación educacional en el mercado laboral.

En síntesis, en una etapa de profundas transformaciones económicas y sociales y bajo un marco de nuevas coordenadas culturales -énfasis en la individualización y desafiliación de instancias intermedias - los jóvenes pobres deben hacer frente el desafío permanente de construir sentidos y elaborar proyectos personales (aunque sean de corto plazo).

Con un acceso a la educación secundaria y niveles de escolarización muy superiores a las de sus padres, paradojalmente, enfrentan mayores dificultades de integración laboral que las generaciones precedentes. Insertos y excluidos a la vez (expresión que da cuenta de la historia personal de educación y trabajo), menos que desarrollar conductas anómicas y generar subculturas segmentadas, en sus prácticas reflejan el sello de la fragilidad de nuestra sociedad y las sombras que evoca la palabra futuro.

Los aspectos aquí reseñados, creemos, delimitan el contexto de desarrollo de la experiencia de vida de los jóvenes adulto sociales insertos en el sistema de educación de adultos. Cerradas las puertas inmediatas para una aventura laboral, conscientes de las consecuencias de quedar pateando piedras (como ya lo han experimentado), el volver a intentarlo significa un esfuerzo personal por construir un proyecto de vida en el marco de la precariedad. Independientemente de su destino final, el reconocimiento de este esfuerzo merece, a lo menos, su consideración analítica.

En lo que resta, se intentará presentar algunos aspectos relevantes del perfil y orientación de estos jóvenes, recogido en el marco del estudio empírico.

\section{Presentación de los PRinCiPales Resultados}

El estudio contempló dos fases complementarias de indagación empírica. En una primera etapa se aplicó un instrumento estructurado a una muestra representativa de jóvenes menores de 24 años cursando su enseñanza secundaria en un Centro de Educación de Adultos. La en-

blecimientos particular subvencionados y un $91 \%$ de establecimientos particulares pagados. Véase, «V Encuesta Nacional a los Actores del Sistema Educativo», CIDE, Santiago, 2004. 
cuesta fue aplicada a una muestra de 381 estudiantes de 18 Centros Integrados de Educación de Adultos de la Región Metropolitana. Junto a esto, se realizaron 10 entrevistas semi-estructuradas a jóvenes que se encuentran cursando su enseñanza media en algunos de estos Centros, intentando profundizar en algunos tópicos relevantes destacados de la encuesta.

Para fines del presente artículo, la información se resumirá en cuatro temas fundamentales; i) perfil general de los jóvenes participantes; ii) educación y trabajo: una nueva extensión de la moratoria; iii) proyecto educativo como desajuste de las expectativas inmediatas $\mathrm{y}$ futuras, $\mathrm{y}$ iv) modelo de acción meritocrático presente en el discurso de los jóvenes. El artículo finalizará con algunas observaciones generales que sirvan de conclusión sobre la realidad de estos jóvenes, y recomendaciones para el sistema educacional a nivel de adultos de acuerdo a los principales hallazgos contenidos en el estudio.

\section{1. ¿Quiénes son los jóvenes adulto sociales?}

Como se ha indicado, el perfil del joven estudiante del sistema de educación de adultos ha sido conceptualizado por las autoridades educativas como joven adulto social. Con esto se hace referencia a una etapa indefinida de estudiante secundario que, sin embargo, se combina permanentemente o en períodos específicos, con actividades laborales y otras responsabilidades familiares y sociales. Se trata, en la mayoría de los casos, de jóvenes en situación de pobreza o de escasos recursos y que presentan rasgos socioculturales propios de la situación de vulnerabilidad social.

En sentido estricto, son jóvenes que vivencian un proceso de redefinición de su propia identidad. La gran mayoría abandonó o fue expulsado del sistema educacional tradicional, y ha permanecido en promedio un período fuera del liceo que se extiende entre 1 y 3 años. Esta situación significa un quiebre con el modelo normativo escolar y, en un sentido profundo, significa un actitud de negación de la identidad estudiantil. Sin embargo, en ese lapso no existen espacios o actividades que reemplacen la totalización simbólica propia del sistema educacional; a diferencia de otras generaciones, el trabajo ya no constituye una alternativa de esa magnitud.

Ante la dificultad de elaborar un proyecto con un mínimo de consistencia y reconociendo las dificultades de integración social a partir

de su actual situación, la finalización de la secundaria emerge como 
una de las pocas alternativas viables y con canales institucionales visibles para estos jóvenes. El retorno a los estudios implica una reelaboración de la condición de estudiantes, incidiendo en la relación que establecen con su entorno, la reorganización de las rutinas y la redefinición del campo de sus obligaciones.

Los testimonios de los jóvenes son elocuentes en resaltar que el volver a cursar la enseñanza media es posible porque en este tipo de centros es donde encuentran un sistema distinto, menos regulado, ${ }^{16} \mathrm{y}$ por tanto, favorable a su acogida.

Volver a intentarlo, también implica un pacto con su entorno familiar más inmediato; como se verá, la gran mayoría de los jóvenes no se dedica a otra actividad durante este período y su sustento depende del conjunto o algún miembro de su grupo familiar cercano.

¿Cuáles son las principales características de los jóvenes? En nuestro estudio, la muestra arrojó un promedio de edad de 19 años, con una alta concentración de jóvenes cuya edad se sitúa entre los 17 y 19 años (el 59.5\% del total). Un tercio de los estudiantes supera los 20 años y en este grupo se concentra un porcentaje importante de mujeres.

En relación al estado civil, existe una preeminencia de solteros, representando el $83 \%$ del total de la muestra. Un análisis por sexo arroja una mayor recurrencia de casados/convivientes entre las mujeres, representando el $22 \%$ de este segmento. En el caso de los hombres, el porcentaje de solteros se eleva al $92.3 \%$ de la muestra. Del total de jóvenes, un $28 \%$ reconoce ser padre o madre, con un alta recurrencia en el caso de las mujeres: el $42,5 \%$ de las encuestadas señala tener a lo menos un hijo, frente al $12,2 \%$ de los estudiantes hombres. La gran mayoría de los jóvenes señala vivir con sus padres o con uno de ellos (cerca del 75\% del total). Solamente en el grupo de las mujeres destaca un $17 \%$ que en la actualidad vive con su pareja.

El ingreso al sistema de educación de adultos de los menores de 24 años responde a algún tipo de problema que dificulta la continuidad de estudios en establecimientos de educación tradicional. La situación de embarazo temprano parece tener incidencia significativa entre las mujeres; entre los hombres, los problemas conductuales, de rendimiento en la experiencia escolar anterior, o la necesidad de tra-

16 Entre los aspectos más destacados por los jóvenes para valorar el centro de educación de adultos se señala la ausencia de normas rígidas de comportamiento, la no exigencia de uniforme para la asistencia a clases, la flexibilidad horaria y un trato mayormente igualitario con los docentes. 
bajar, ha incidido en una trayectoria irregular, y el abandono del liceo por un tiempo indefinido.

Dentro de los datos disponibles en la encuesta, puede señalarse que la mayoría de los jóvenes se incorporó recientemente al establecimiento educacional de adultos: cerca de un $40 \%$ del total de los encuestados asiste a clases en el Centro desde hace un año y un $47 \%$ se incorporó al mismo en el presente año escolar. Solamente un 13\% del total de encuestados reconoce permanecer hace dos años o más en el mismo establecimiento. Como se ha indicado, en términos generales la principal causa de abandono del liceo es el fracaso escolar, sin embargo, este hecho está asociado a un conjunto de variables íntimamente relacionadas y que dicen relación con una frágil base de apoyo social para estos jóvenes. La perspectiva de egreso definitivo en el sistema de educación de adultos es también incierta: cerca de un $30 \%$ de los estudiantes abandonan anualmente sus estudios en esta modalidad.

La información proporcionada sobre convivencia familiar, indica un alto porcentaje de jóvenes que vive con uno o ambos padres; resulta significativa la valoración que perciben de su entorno cercano a sus actividades educativas; cerca de tres cuartos de los consultados señala que en casa reciben apoyo o mucho apoyo a su situación actual de estudiantes. No se observa, desde ese punto de vista, un cuadro de crisis o abandono familiar a partir de las respuestas recogidas en la indagación.

Sin embargo, esta constatación puede ser relativizada a partir de la profundización de la experiencia familiar de los sujetos. Menos que la reproducción de un modelo familiar tradicional, en la historia de vida de los jóvenes existe una gran heterogeneidad de experiencias. Recurrentemente, muchos de los entrevistados remiten a algún referente cercano como su apoyo personal por sobre el grupo familiar; éstos pueden ser muy disímiles (abuelos, tíos, etc.) pero que, de igual manera, sirven de soporte a su actual situación de estudiantes y representan un marco de referencia afectivo que incide en una cierta estabilidad personal, independiente de su edad actual, trayectoria y opciones de vida.

Finalmente, la situación socioeconómica de los jóvenes presenta altos niveles de homogeneidad; cerca del $60 \%$ se encuentra bajo la línea de pobreza y la gran mayoría manifiesta que sus familias tienen dificultades económicas importantes. Pese a este cuadro general de carencias, el grueso de los jóvenes dedica su tiempo básicamente a asistir al centro de estudios y depende económicamente de su familia; 
el $60 \%$ de los encuestados señala no trabajar en la actualidad. El siguiente cuadro resume las principales características aquí reseñadas.

\section{Cuadro 1 \\ Perfil joven adulto social}

\begin{tabular}{|l|l|}
\hline Edad & $\begin{array}{l}\text { Jóvenes de ambos sexos, promedio de edad 19 años; 20\% de } \\
\text { los hombres y 31\% de las mujeres por sobre los } 20 \text { años. }\end{array}$ \\
\hline Experiencia escolar & $\begin{array}{l}\text { Desertores del sistema escolar tradicional entre 1 y 3 años, } \\
\text { en la actualidad cursando diversas modalidades de forma- } \\
\text { ción ofrecidas en centros de educación de adultos. }\end{array}$ \\
\hline $\begin{array}{l}\text { Causa de la } \\
\text { deserción escolar }\end{array}$ & $\begin{array}{l}\text { Rendimiento escolar, problemas conductuales, embarazo o } \\
\text { problemas económicos. }\end{array}$ \\
\hline $\begin{array}{l}\text { Estado civil } \\
\text { y situación actual }\end{array}$ & $\begin{array}{l}\text { Alto porcentaje de solteros, viviendo en casa de sus padres o } \\
\text { de uno de ellos; } 28 \% \text { reconoce tener hijos; mayor incidencia } \\
\text { en el caso de mujeres (42,5\%). }\end{array}$ \\
\hline Percepción de apoyo & $\begin{array}{l}\text { Percepción favorable de apoyo familiar a su actividad, } \\
\text { aunque en muchos casos, referido a un miembro de la fami- } \\
\text { lia extensa. Dependencia económica de un integrante o del } \\
\text { conjunto de su entorno familiar inmediato. }\end{array}$ \\
\hline $\begin{array}{l}\text { Situación } \\
\text { socioeconómica }\end{array}$ & $\begin{array}{l}\text { Gran homogeneidad socioeconómica; 60\% de los jóvenes } \\
\text { bajo línea de pobreza. }\end{array}$ \\
\hline $\begin{array}{l}\text { Elementos } \\
\text { de identidad }\end{array}$ & $\begin{array}{l}\text { Rechazo a modelo tradicional de estudiante y valoración de } \\
\text { sistema de educación de adultos en función a flexibilización } \\
\text { normativa. }\end{array}$ \\
\hline
\end{tabular}

\section{Trabajo y estudio: una nueva moratoria}

La gran mayoría de los jóvenes ha tenido algún tipo de experiencia laboral. En el estudio, solamente un $19 \%$ de los entrevistados señala no haber trabajado anteriormente. Ante diferentes alternativas, existe un grupo que manifiesta haberlo hecho en períodos acotados, por lo general, en vacaciones, mientras que alrededor de un $40 \%$ manifiesta haberlo hecho mientras estudiaba o en el período en que había abandonado los estudios formales.

En el momento de la investigación, un tercio de los entrevistados señala que combina su experiencia educativa con alguna actividad laboral; aunque el grueso de estos jóvenes lo hace en actividades esporádicas, por lo general de carácter independiente y destinando una cantidad de horas reducidas a esta actividad.

Tanto en el período en que no asistieron regularmente a clases como en el actual momento como estudiantes de la educación de adultos, estos jóvenes tienen una experiencia fragmentaria con el 
mundo del trabajo. La gran mayoría de ellos sólo ha trabajo en períodos cortos de tiempo, en actividades de muy baja calificación (actividades de servicio o trabajo por cuenta propia) y percibiendo salarios reducidos. Del conjunto de los jóvenes que manifiesta haber tenido una experiencia laboral, la mitad lo ha hecho como trabajador por cuenta propia o desempeñando alguna tarea de servicios en el comercio.

Lo anterior no inhibe una actitud positiva hacia el trabajo y una predisposición favorable a la integración laboral, independientemente de las condiciones materiales y salariales; los jóvenes entrevistados manifiestan una actitud abierta hacia las posibilidades de trabajo realmente existentes y reconocen que en sus actuales condiciones, la experiencia laboral puede ser útil como forma de aprendizaje, desarrollo personal y resolución de necesidades básicas.

Pese a esto, como se ha indicado, dos tercios de los jóvenes entrevistados manifiesta no trabajar en la actualidad. ¿A qué se debe esta situación?

La valoración general de la experiencia laboral y la disposición a integrarse al mundo del trabajo sólo se ve amagada, en el discurso de los jóvenes, por el peso que ellos le asignan a la experiencia educativa y el rol fundamental que tienen los estudios para acceder a un puesto de trabajo y lograr ser alguien en la vida. El 70\% de los entrevistados señala que es necesario tener la licencia secundaria para poder acceder a un puesto de trabajo mayormente estable y más del $60 \%$ reconoce que sólo los estudios post-secundarios aseguran una buena inserción laboral.

Desde nuestro punto de vista, en un sentido opuesto a la tendencia que caracterizó el sistema de educación de adultos hasta hace pocos años, estos jóvenes reactualizan un modelo de moratoria propia del sistema de educación tradicional debido a que el mundo del trabajo está significativamente cerrado para ellos. En sentido estricto, su nuevo pacto educativo (con su familia y ellos mismos) implica la postergación del ingreso a un mundo laboral que sólo le ofrece integración precaria, y la dedicación por tiempo completo a sus estudios. Aunque las exigencias académicas y horarias son menores al sistema tradicional, en el discurso de los jóvenes prevalece el modelo de moratoria y, como se verá, la definición de expectativas a futuro fundada en el modelo de carrera educacional. 


\section{Proyecto educativo como desajuste de expectativas}

El grueso de los jóvenes manifiesta interés por continuar sus estudios una vez obtenida la licencia media en el centro de educación de adultos. Entre las distintas posibilidades, la mayoría prioriza alguna carrera universitaria, aunque la formación técnica intermedia también es una opción para un segmento relevante de ellos (el 47,5\% de los jóvenes encuestados señala que le gustaría estudiar una carrera universitaria, mientras que el $37,5 \%$ se inclina por una carrera técnico profesional). Pese a lo anterior, esta intención se presenta de un modo general sin que exista, necesariamente, un proyecto predefinido, un área de interés o un conocimiento cabal de las características, condiciones y obligaciones que implica tal hecho.

Interrogados sobre opciones específicas de interés, por lo general las referencias son a profesiones tradicionales o ámbitos muy generales, sin que exista claridad sobre oferta educativa o campo laboral específico.

En otro sentido, existe un bajo interés entre los jóvenes por aprender un oficio en un curso de corta duración, similar al modelo predominante de formación para el trabajo que ha caracterizado los programas gubernamentales en los últimos años, ${ }^{17}$ y que justamente, identifica como población objetivo a jóvenes con un perfil socioeconómico como el de estos estudiantes.

Desde nuestro punto de vista, el sistema escolarizante tradicional con muy débiles capacidades de diversificación, incide de manera importante en esta orientación subjetiva. En efecto, en el sistema de educación de adultos las propuestas curriculares, modalidad de enseñanza y estrategia de evaluación no difieren significativamente del modelo predominante en la educación chilena, extremadamente rígido en relación a perfilar una estrategia orientada hacia estudios post-secundarios de tipo universitario. De hecho, la gran mayoría de los establecimientos de educación de adultos en nuestro país que imparten enseñanza media, se acogen a modalidades curriculares de educación científico humanista, mayormente distante de la inserción temprana en el mundo del trabajo y con un énfasis en la formación académica tradicional. ${ }^{18}$

17 Nos referimos al Programa Chile Joven, iniciativa gubernamental durante los noventa destinado a otorgar capacitación laboral a jóvenes en situación vulnerable con perspectivas de una mejor integración laboral.

18 Esta tendencia parece ser revertida en las nuevas propuestas curriculares y de organización institucional generadas en el marco del Programa Chile Califica, que recientemente comienzan a implementarse. 
La ausencia de una propuesta formativa que ayude a perfilar un ámbito o área de interés de desarrollo profesional inmediato incrementa la moratoria y, a manera de círculo vicioso, constituye al centro de educación de adultos en un espacio de refugio para muchos de estos jóvenes. Lo anterior, por cierto, puede ser favorable como mecanismo de generación de lazos de sociabilidad y confianza, pero, al mismo tiempo, creemos, genera un efecto de desajuste de expectativas con consecuencias negativas una vez cumplido el objetivo de la certificación secundaria. En este sentido, no debería ser sorprendente que un joven egresado de la enseñanza media de este sistema (con más de 21 años cumplidos) se pregunte y ahora, ¿qué hacer?

\section{Preeminencia de un modelo meritocrático}

Muy vinculado a lo anterior, en nuestro estudio se intentó indagar acerca de las orientaciones de acción que los jóvenes privilegian en diversos ámbitos o situaciones.

En términos generales, independientemente de un ejercicio de evaluación de la rentabilidad de la licencia secundaria, los jóvenes apuestan (con mayor o menor nivel de objetividad) a la acumulación de capital cultural para hacer frente de mejor manera y con mayores recursos, el mundo del trabajo. Pero este reconocimiento del valor de los estudios, como hemos visto, constituye una afirmación muchas veces formal, no necesariamente traducible en acción concreta una vez egresados del establecimiento en que actualmente se encuentran. Es por esto que junto al discurso de valor educacional, se releva de manera muy particular el principio de esfuerzo y espíritu de superación entendido como una cuestión actitudinal frente a los desafíos cotidianos.

Por cierto, lograr finalizar los estudios es parte de esta entrega y dedicación, pero además, el esfuerzo personal es un mecanismo que, aunque no se logren las metas de estudio, también puede llevar a triunfar en la vida. El 80\% de los encuestados estuvo de acuerdo o muy de acuerdo con la afirmación «si pones empeño en tu trabajo, aunque no estudies después de cuarto medio, igual puedes surgir».

La orientación meritocrática está presente de manera consistente en el discurso de estos jóvenes, constituyendo, a no dudarlo, su principal capital. Por ejemplo, consultados ante la eventualidad de búsqueda de trabajo, un porcentaje importante se inclina por recurrir a mecanismos formales tradicionales (búsqueda de aviso en el diario, $41,5 \%$ ) por sobre los mecanismos informales de redes de intermedia- 
ción (conocido como pituto, 24,1\%). Este último aspecto, recurrir a terceros obviando mecanismos formales y estandarizados de acceso a puestos de trabajo es, por lo general, mucho más destacado en mediciones de la orientación laboral de otros actores. Una hipótesis posible frente a esta situación radicaría en una escasa densidad de redes sociales de los jóvenes, aspectos que los llevaría a desconocer la importancia de los mecanismos informales.

Sin embargo esta hipótesis parece poco plausible, más aún si la orientación general hacia el mundo del trabajo y sus mecanismos de integración encuentra en el discurso de los jóvenes una aceptación acrítica. ${ }^{19}$ En diversos contextos situacionales los jóvenes parecen reconocer las reglas del juego y fundar sus posibilidades a partir de los recursos que tienen al alcance. Como hemos insistido, tal actitud dista bastante de convertirse en una conducta extra sistémica, acercándose más bien a un modelo propiamente de reproducción social.

El peso desmedido de la argumentación meritocrática puede ejemplificarse con los aspectos privilegiados por los jóvenes cuando se les consulta acerca de las variables que identifican un buen rendimiento en el lugar de trabajo. Al solicitárseles que señalaran los tres conceptos más relevantes que prioriza un empleador al contratar a una persona, los encuestados se inclinan, fundamentalmente, por aspectos actitudinales tales como la responsabilidad, la puntualidad y la honradez.

Esta opción advierte sobre el marcado peso de estos aspectos en la organización del proyecto de mediano plazo de los jóvenes, y la minimización de otras dimensiones tan importantes como son las habilidades específicas y los conocimientos generales.

En efecto, dentro de un conjunto de conceptos propuestos para su identificación, aquellos que refieren a competencias generales de base (cuestión que ha sido destacado en los discursos educativos de los últimos años), logran un bajo o nulo reconocimiento entre los jóvenes consultados. Es el caso de las competencias de pensamiento lógico (saber matemáticas) y comunicacionales (saber leer y escribir, hablar

19 Esta orientación contrasta con los resultados obtenidos a la misma pregunta en estudiantes secundarios del sistema formal. Consultados sobre los mecanismos de búsqueda y obtención de trabajo, la encuesta arrojó que un 47,7\% de los estudiantes secundarios buscaría algún pituto o contacto, un $29,5 \%$ optó por la alternativa de buscar avisos en los diarios, el 19,9\% iría a dejar un currículo a personas conocidas, el 2,9\% dice que recurriría a una municipalidad, y un 4,5\% señaló otra opción (que por lo general se relacionó con las anteriores). Véase Álvarez, Ibáñez y Sepúlveda; 2000. 
bien). También logra un muy bajo reconocimiento la capacidad de análisis, la creatividad y el uso y manejo de tecnología comunicacional.

Este último aspecto es la cara crítica de la actitud integrativa de los jóvenes; al relevar exclusivamente un campo de las capacidades necesarias para integrarse adecuadamente al mundo del trabajo (lo actitudinal), sus posibilidades pueden verse fuertemente restringidas, frustrando, en el corto plazo los sueños y expectativas que sostiene su permanencia en el sistema educacional y el conjunto de valores ya destacados.

El siguiente cuadro sintetiza las opciones marcadas por los jóvenes encuestados.

\section{Cuadro 2}

Principales aspectos que destacaría un empleador para contratar a una persona

\begin{tabular}{|c|c|c|c|c|c|}
\hline Responsabilidad & $74 \%$ & Puntualidad & $39.9 \%$ & $\begin{array}{c}\text { Ser } \\
\text { organizado }\end{array}$ & $9.4 \%$ \\
\hline Buena presencia & $31 \%$ & $\begin{array}{c}\text { Resolución } \\
\text { de problemas }\end{array}$ & $2.1 \%$ & $\begin{array}{l}\text { Saber trabajar } \\
\text { en grupo }\end{array}$ & $8.7 \%$ \\
\hline $\begin{array}{l}\text { Capacidad } \\
\text { de análisis }\end{array}$ & $3.7 \%$ & Hablar bien & $9.2 \%$ & Creatividad & $5.5 \%$ \\
\hline Saber matemáticas & $0.8 \%$ & $\begin{array}{c}\text { Saber tomar } \\
\text { decisiones }\end{array}$ & $7.6 \%$ & $\begin{array}{l}\text { Saber usar } \\
\text { tecnología como } \\
\text { computador }\end{array}$ & $10.5 \%$ \\
\hline $\begin{array}{c}\text { Espíritu } \\
\text { de superación }\end{array}$ & $13.4 \%$ & Honradez & $20.2 \%$ & $\begin{array}{l}\text { Saber leer } \\
\text { y escribir }\end{array}$ & $10.5 \%$ \\
\hline $\begin{array}{c}\text { Entender cómo } \\
\text { funciona una empresa }\end{array}$ & $9.4 \%$ & $\begin{array}{l}\text { Manejo } \\
\text { de grupo }\end{array}$ & $0.3 \%$ & Autocontrol & $3.7 \%$ \\
\hline
\end{tabular}

Este cuadro general de priorizaciones no resulta demasiado distante de lo observado en los jóvenes estudiantes de la enseñanza media en el sistema formal. Como puede verse en el siguiente cuadro comparativo, los aspectos priorizados por los estudiantes del sistema de educación de adultos no se diferencian mayormente de lo que relevan jóvenes que estudian en establecimientos municipales, particular subvencionados o corporaciones, tanto a nivel científico humanista como técnico profesional.

La gran diferencia (la brecha) aparece en el caso de los estudiantes de establecimientos particulares pagados los que, pese a destacar también la dimensión actitudinal como prioritaria, equilibran sus opciones con conocimientos (saber leer y escribir, saber matemáticas) y 
habilidades (saber tomar decisiones). Paradojalmente, son estos jóvenes los que mayoritariamente postergarán su entrada al mundo del trabajo y quienes estudiarán en la educación superior. ${ }^{20}$

\begin{tabular}{|c|c|c|c|c|}
\hline \multicolumn{5}{|c|}{ Tipo de Establecimiento } \\
\hline Municipal & $\begin{array}{c}\text { Particular } \\
\text { subvencionado }\end{array}$ & Particular & Corporación & $\begin{array}{c}\text { Centro educa- } \\
\text { ción adultos }\end{array}$ \\
\hline Responsabilidad & Responsabilidad & Responsabilidad & $\begin{array}{c}\text { Responsabili- } \\
\text { dad }\end{array}$ & Responsabilidad \\
\hline $\begin{array}{c}\text { Buena } \\
\text { presencia }\end{array}$ & $\begin{array}{c}\text { Buena } \\
\text { presencia }\end{array}$ & $\begin{array}{c}\text { Buena } \\
\text { presencia }\end{array}$ & $\begin{array}{c}\text { Buena } \\
\text { presencia }\end{array}$ & Puntualidad \\
\hline Puntualidad & Puntualidad & Honradez & Honradez & $\begin{array}{c}\text { Buena } \\
\text { presencia }\end{array}$ \\
\hline Honradez & Honradez & $\begin{array}{c}\text { Saber leer y } \\
\text { escribir }\end{array}$ & Puntualidad & Honradez \\
\hline $\begin{array}{c}\text { Espíritu de } \\
\text { superación }\end{array}$ & $\begin{array}{c}\text { Espíritu de } \\
\text { superación }\end{array}$ & $\begin{array}{c}\text { Saber tomar } \\
\text { decisiones }\end{array}$ & $\begin{array}{c}\text { Espíritu de } \\
\text { superación }\end{array}$ & $\begin{array}{c}\text { Espíritu de } \\
\text { superación }\end{array}$ \\
\hline
\end{tabular}

Sin ánimos de mayor desarrollo, podría decirse que este ámbito temático constituye un indicador que remite a la gran diferenciación en el sistema educacional chileno, reflejando, sin duda, la honda diferenciación en la calidad, aspiración y posibilidad de desarrollo futuro de los jóvenes participantes en cada uno de los niveles y, por cierto, muy particularmente los jóvenes adulto sociales.

Proyectándose en el futuro, estos jóvenes apuestan por una situación personal mejor y como se ha insistido en estas páginas, la base de este sueño lo constituye la educación recibida y el propio esfuerzo personal por lograrlo (casi el $90 \%$ de los encuestados cree que en el futuro le irá mejor que en la actualidad).

En los márgenes de la exclusión social y con una muy baja prospectiva de éxito futuro, discursivamente estos jóvenes recurren a los argumentos más tradicionales de la sociedad integradora; en su discurso existe una escasa consideración de apoyo en redes sociales horizontales o verticales (lo que refuerza la percepción de extremada precariedad) y no se reconocen mínimos trazados una vez cumplida la meta de lograr la certificación de enseñanza media.

Con todo, el solo hecho de estar ahí y la decisión de un nuevo intento de estos jóvenes por surgir en el marco de la educación de adultos, es una señal poderosísima de las características y desafíos de

20 Véase Martinic y Sepúlveda, 2002. 
la sociedad chilena actual que, visiblemente fragmentada, sin embargo logra sostener bases de integración ineludibles. De esa dimensión es el desafío educacional en el futuro inmediato.

\section{CONCLUSIONES}

Sin ánimos de reiterar las afirmaciones sostenidas en las páginas precedentes, es posible sostener que los principales aspectos relevados en este artículo remiten a los siguientes aspectos.

i) Emergencia del joven adulto social. Como se ha indicado, la figura de jóvenes adulto sociales, esto es, jóvenes que habiendo abandonado la enseñanza secundaria, retornan a completar su licencia en el contexto del sistema de la educación de adultos, constituye una realidad relativamente nueva en nuestra sociedad y responde a varios factores asociados a la transformación del mundo del trabajo y las exigencias de escolarización. En este plano, lo más relevante tiene que ver con la restricción del mercado laboral para las generaciones jóvenes y la exigencia creciente de certificación secundaria (como exigencia mínima) en la mayoría de los trabajos, independientemente del nivel de competencias requeridas.

Los jóvenes objeto de esta investigación, comparten una racionalidad social fuertemente instalada: la licencia secundaria se constituye en un factor fundamental de integración. Por cierto, tener estudios secundarios completos no asegura, ni con mucho, el acceso a un empleo - sin hacer, siquiera, la distinción entre empleo decente o nopero no poseer estudios secundarios completos significa cercenar significativamente las posibilidades de acceso al trabajo.

Como se ha señalado, esta constatación es ampliamente compartida y constituye el sentido fundamental de acción de los jóvenes en todo nivel. En rigor, esta práctica constituye un indicador de la perspectiva de integración presente en la práctica juvenil y los esfuerzos significativos por salir adelante que esta orientación contiene.

ii) Estructura familiar, afecto y presencia de adultos cercanos. Pese a que muchos de ellos han tenido un período prolongado fuera del sistema escolar, y una experiencia relativa en el mundo del trabajo, la mayoría de los jóvenes descansan en el sustento que reciben de su entorno familiar cercano. Un grueso de ellos reconoce apoyo a su tarea de estudiante tanto en el plano afectivo, como en el plano material. 
Este cuadro general, con todo, debería ser matizado en función de los resultados obtenidos en la indagación cualitativa. Como se señaló, el entorno familiar de los jóvenes presenta rasgos de fragmentación y carencias marcadas en el plano afectivo. La incidencia de la ruptura familiar parece importante, y es sustituida (no siempre de manera adecuada) por la existencia de apoyos de otros familiares (en general, abuelos y tíos).

El espacio educativo es valorado en este contexto de déficit afectivo, y muchos de los estudiantes reconoce la acogida que encuentran entre compañeros y profesores, independientemente de los aspectos propiamente formativos.

iii) Trayectoria laboral marginal y débil inserción en el mercado de trabajo. De la indagación realizada, destaca el hecho que la mayoría de los jóvenes estudiantes han tenido experiencia en algún empleo. Sin embargo, como se señaló, ésta es mayoritariamente marginal y se concentra en oficios de baja calificación, escaso salario y corta duración. La mayoría de los jóvenes participantes no reconocen aprendizajes significativos de esta experiencia siendo limitado, por lo mismo, el desarrollo de una cultura laboral propiamente tal.

La fragilidad de la experiencia laboral redunda en una decisión significativa: no volver al trabajo hasta concluir la enseñanza secundaria. En la mayoría de los discursos, esta decisión se asocia a la expectativa de un mejor trabajo futuro, aunque éste resulte de difícil objetivación.

iv) Precariedad de redes sociales. En términos generales los jóvenes carecen de redes sociales significativas que ayuden a la búsqueda de trabajo presente o futura. De hecho, un porcentaje mayoritario de los jóvenes estudiados señala que privilegiarían mecanismos formales de búsqueda de empleo, por sobre la recurrencia a contactos $\mathrm{u}$ otros medios informales de acceso. Como se indicó, esta perspectiva contrasta significativamente con los resultados obtenidos en otros estudios dirigidos a jóvenes.

Los jóvenes dependen en buena medida de su entorno familiar, pero éste presenta rasgos de precariedad bastante homogéneos, no siendo una alternativa adecuada para resolver problemas como el de alcanzar un puesto de trabajo.

v) Preeminencia de modelo meritocrático para el alcance de logros. Muy vinculado a lo anterior, los jóvenes rescatan - por lo menos discursivamente- el valor del esfuerzo individual y el mérito en las actividades realizadas como el mecanismo básico para surgir y 
salir adelante. En la gran mayoría de los casos, la valoración del esfuerzo individual (que, como vimos, resulta funcional al modelo cultural predominante) lleva a formular un imagen de futuro optimista, de superación de sus padres o adultos cercanos y de realización de expectativas de felicidad. Pese a lo anterior, esta formulación remite mayormente al ámbito de la convivencia y las relaciones sociales y pierde fuerza respecto a la configuración de un proyecto laboral sistemático. $^{21}$

vi) Expectativas formativas post-secundarias. La mayoría de los entrevistados manifiesta el deseo o la expectativa de continuar estudiando una vez egresados de la enseñanza secundaria, cursando, en lo posible, una carrera universitaria. En la mayoría de los casos, esta manifestación de interés no encuentra un conducto con visos de viabilidad, elaborándose expectativas desmedidas o precariamente fundadas. Como se intentó argumentar, detrás de esta disociación puede existir un implícito reconocimiento de irrealización, produciéndose, más bien, la reproducción mecánica de un discurso modelístico.

Lo anterior estaría asociado a la debilidad para elaborar alternativas de salida del sistema (donde la formación ofrecida también presentaría altos niveles de restricción), generándose una suerte de gran vacío (nuevamente) al término de la licencia secundaria.

vii) Valoración de espacio educacional alternativo pero bajo reconocimiento de recursos necesarios para hacer frente desafios futuro inmediato. Como se indicó, la gran mayoría de los jóvenes participan de un modelo de extensión de la moratoria debido a las precarias condiciones de integración al mundo del trabajo. En este plano, los jóvenes valoran y reconocen la importancia de este sistema de enseñanza, reconociendo que no sería posible re-integrarse en el modelo tradicional más exigente y mayormente reglamentado.

Sin embargo, los antecedentes indican que el nivel de adquisición de conocimientos y habilidades en general es bajo, representando un aporte marginal una vez egresados y obtenida su licencia secundaria

21 Este aspecto ha sido ampliamente desarrollado en estudios sobre la orientación discursiva de jóvenes pobres en países desarrollados. En éstos se destaca que la gran mayoría de los jóvenes son conscientes de las dificultades estructurales existentes para alcanzar sus metas, pero, pese a ello, su actitud es básicamente optimista, basada en la confianza en sus propias capacidades para alcanzar objetivos y lograr proyectos de vida. Como contraste, la desorientación y la prolongación de las decisiones constituye el principal sello generacional. Véase Schneider y Stevenson, 1999. 
(de hecho, un grupo no menor reconoce que están ahí básicamente por el objetivo pragmático de este esfuerzo).

Muy ligado al énfasis meritocrático de su discurso, los jóvenes resaltan los valores actitudinales para el éxito en el mundo del trabajo en el futuro inmediato (honradez, puntualidad, responsabilidad). Esta perspectiva minimiza aquellos aspectos propios de la sociedad del conocimiento. En este sentido, el capital humano relevado presenta un fuerte desnivel, aspecto que, sin duda implica una desventaja en el mercado laboral.

En síntesis, en este estudio hemos intentado demostrar que la experiencia y el discurso de los jóvenes parece expresar, vívidamente, el complejo proceso de inclusión/exclusión que caracteriza buena parte de nuestra vida social.

Reducidos los mecanismos formales de integración laboral y relegados a alternativas de bajo retorno en el desarrollo de su escolarización, estos jóvenes reelaboran proyectos personales y se esfuerzan por surgir en condiciones de gran precariedad. Su recurso principal radica, básicamente, en el esfuerzo individual al carecer de redes mínimas de apoyo.

Sus sueños son, muchas veces, la escenificación de un modelo predominante en la sociedad, pero de la que ellos no forman parte o tienen escasas posibilidades de acceso. Como contrapartida, no parecen existir mecanismos o instancias institucionales reconocidas que sirvan como vehículo de canalización de este esfuerzo personal.

Una mayor preocupación por este desajuste o disociación cultural permitiría un mejor aprovechamiento de las energías involucradas en esta tarea cotidiana, proyectando, al mismo tiempo, un escenario social más optimista que el que se perfila a la luz de la experiencia de estos jóvenes y las actuales respuestas que encuentran en la sociedad.

Desde este punto de vista, el sistema de educación de adultos contiene el desafío no sólo de acoger a jóvenes que buscan una nueva oportunidad de completar su enseñanza secundaria, sino que favorecer estrategias funcionales a la formación profesional temprana, la diversificación de ofertas educativas, desbloqueando el modelo prouniversitario que caracteriza el conjunto del sistema educacional chileno, y equilibrando los criterios de desarrollo personal, fortaleciendo objetivos y metodologías que permitan la competencia de los sujetos en escenarios fuertemente segmentados. 
La decisión de estos jóvenes de volver a intentarlo, merece un esfuerzo institucional de esa magnitud.

SANTIAGO (CHILE), NOVIEMBRE 2004

RECIBIDO: NOVIEMBRE 2004

ACEPTADO: DICIEMBRE 2004

\section{BIBLIOGRAFíA}

Álvarez, C.; S. IbÁÑEZ y L. SEPÚlVEda (2000): «Estrategia de búsqueda de empleo de los jóvenes estudiantes secundarios de la Región Metropolitana». Documento $N^{o} 4$, Volumen II. Santiago: CIDE.

ASESorías PARA El Desarrollo (2002): «Diagnóstico de los centros de educación integrada de adultos». Informe 2. Santiago: Asesorías para el Desarrollo.

BALANDier, G (1975): Antropo-lógicas. Barcelona: Península.

BELLEÏ, C. (2000): «Educación media y juventud en los 90: actualizando la vieja promesa». Última Década $\mathrm{N}^{\circ} 12$. Viña del Mar: Ediciones CIDPA.

CELADE (2000): Juventud, población y desarrollo: problemas, posibilidades y desafios. Santiago: CELADE-FNUAP.

CEPAL (2000): La brecha de la equidad: una segunda evaluación. Santiago: CEPAL.

CIDE (2004): «V Encuesta Nacional a los Actores del Sistema Educativo». Santiago: CIDE.

Cox, C. (2001): «Políticas educacionales y procesos de cambio en la educación media de Chile en los años noventa». En C. BRASLAVSKY (org.): La educación secundaria ¿cambio o inmutabilidad? Análisis y debate de procesos europeos y latinoamericanos contemporáneos. Buenos Aires: Santillana.

ESPINOZA, V. et al. (2000): «Ciudadanía y juventud. Análisis de los perfiles de oferta y demanda de las políticas sociales ante la nueva realidad juvenil». Santiago: Fondo para el Estudio de las Políticas Públicas.

MAFFESOLI, M. (1993): El conocimiento ordinario. México: FCE.

MARTINIC, S. y L. SEPÚlvedA (2002): «La enseñanza secundaria ¿prepara para el trabajo?». Revista Chilena de Antropología $\mathrm{N}^{\circ} 16$. Santiago: Universidad de Chile.

RodrígueZ, E. (2000): «Juventud y desarrollo en América Latina: desafíos y prioridades en el comienzo de un nuevo siglo». Seminario La Renovación del Capital Humano y Social, Nueva Orleáns; Cuadragésima Reunión Anual de la Asamblea de Gobernadores de Banco Interamericano de Desarrollo.

RoDRIK, D. (2001): «¿Por qué hay tanta inseguridad económica en América Latina?». Revista de la CEPAL $\mathrm{N}^{\circ} 73$. Santiago: CEPAL.

SchneIder, B. y D. Stevenson (1999): «The Ambitious Generation: America's teenagers; motivated but directionless». New Haven, Yale University Press.

Silva, J. C. (2002): «Juventud y tribus urbanas: en busca de identidad». Última Década $\mathrm{N}^{\circ} 17$. Viña del Mar: Ediciones CIDPA.

VALENZUELA, E. (1984): La rebelión de los jóvenes. Santiago: Ediciones SUR.

WeInSteIn, J. (1988): Los jóvenes pobladores en las protestas nacionales (19831984). Una visión sociopolítica. Santiago: CIDE.

Wormald, G. y J. Ruiz-TAgLE (1999): «Exclusión social en el mercado del trabajo. El caso de Chile». Documento de Trabajo Nº105. Santiago: OIT, Fundación Ford. 